Canadian

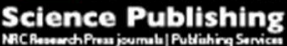

Biochemistry and Cell Biology Biochimie et biologie cellulaire

\title{
Role of STARD4 and NPC1 in intracellular sterol transport
}

\begin{tabular}{|r|l|}
\hline Journal: & Biochemistry and Cell Biology \\
\hline Manuscript ID & bcb-2015-0154.R1 \\
\hline Manuscript Type: & Invited Review \\
\hline Date Submitted by the Author: & $19-J a n-2016$ \\
\hline Complete List of Authors: & $\begin{array}{l}\text { Maxfield, Frederick; Weill Cornell Medical College, Biochemistry } \\
\text { Iaea, David; Weill Cornell Medical College, } \\
\text { Pipalia, Nina; Weill Cornell Medical College, Biochemistry }\end{array}$ \\
\hline Keyword: & Cholesterol, STARD4, lipoproteins, Niemann-Pick C, lipid transport proteins \\
\hline &
\end{tabular}




\section{Role of STARD4 and NPC1 in intracellular sterol transport}

Frederick R. Maxfield, David B. Iaea, and Nina H. Pipalia

Department of Biochemistry

Weill Cornell Medical College

New York, NY 10065 USA

Corresponding author

Frederick R. Maxfield

Department of Biochemistry

Weill Cornell Medical College

1300 York Avenue

New York, NY 10065

Tel: 2127466405

Fax: 2127468875

Email: frmaxfie@med.cornell.edu 


\begin{abstract}
Cholesterol plays an important role in determining the biophysical properties of membranes in mammalian cells, and the concentration of cholesterol in membranes is tightly regulated. Cholesterol moves among membrane organelles by a combination of vesicular and nonvesicular transport pathways, but the details of these transport pathways are not well understood. In this review, we discuss the mechanisms for nonvesicular sterol transport with an emphasis on the role of STARD4, a small, soluble, cytoplasmic sterol transport protein. STARD4 can rapidly equilibrate sterol between membranes, especially membranes with anionic lipid headgroups. We also discuss the sterol transport in late endosomes and lysosomes, which is mediated by a soluble protein, NPC2, and a membrane protein NPC1. Homozygous mutations in these proteins lead to a lysosomal lipid storage disorder, Niemann-Pick disease type C. Many of the disease causing mutations in NPC1 are associated with degradation of the mutant NPC1 proteins in the endoplasmic reticulum. Several histone deacetylase inhibitors have been found to rescue the premature degradation of the mutant NPC1 proteins, and one of these is now in a small clinical trial.
\end{abstract}

Key words

Cholesterol, STARD4, lipoproteins, lipid transport proteins, Niemann-Pick C 


\section{Introduction: Distribution and transport of sterols in mammalian cells}

There are two main sources of cholesterol in most nucleated mammalian cells. As illustrated in Figure 1, low density lipoproteins (LDL) bind to LDL-receptors (LDL-R) and are internalized by clathrin mediated endocytosis into early/sorting endosomes (SE), where LDL dissociates from the LDL-R due to the acidic pH (Brown et al. 1983; Yamashiro and Maxfield 1984). The LDL-R are recycled back to the plasma membrane (PM), while the LDL is delivered to late endosomes (LE) by maturation of the SE (Dunn and Maxfield 1992; Ghosh et al. 1994). The core of the LDL, which is $\sim 90-95$ mol\% cholesteryl ester (CE), is delivered to LE/Ly and hydrolyzed to free cholesterol by lysosomal acid lipase (LAL) (Brown et al. 1975; Van Berkel et al. 1975). A sterol transport protein, NPC2 (Friedland et al. 2003), transports newly generated free cholesterol to the N-terminal domain (NTD) of the NPC1 protein residing in limiting membrane of lysosomes (Infante et al. 2008). NPC1 is 1278 amino acid cysteine rich glycoprotein, with 13 transmembrane domains, five of which are sterol sensing domains (SSD) (Kwon et al. 2009; Scott and Ioannou 2004). Cholesterol delivered to NTD of NPC1 is then effluxed out of the LE/Ly by a mechanism that is not well understood but may involve delivery to the limiting membrane of the organelle followed by transport to other organelles. Cholesterol is also synthesized in most nucleated mammalian cells by a multistep process that takes place mainly in the endoplasmic reticulum (ER).

The ER is also the main organelle for sensing and regulating cellular cholesterol levels (Brown and Goldstein 1997; Goldstein and Brown 2001; Radhakrishnan et al. 2008). The transcriptional regulator, sterol response element binding protein (SREBP2), is synthesized as a transmembrane protein that is held in the ER in complex with a chaperone protein, SCAP, which binds to an ER resident protein, INSIG. When cellular cholesterol levels are decreased, SCAP 
and SREBP2 dissociate from INSIG and move to the Golgi apparatus in transport vesicles. SREBP2 is cleaved in the Golgi by two proteolytic steps that release the mature, soluble transcription factor that translocates to the nucleus to initiate transcription of genes involved in cholesterol synthesis and uptake (Brown and Goldstein 1997; Radhakrishnan et al. 2008). In a separate regulatory process, the ER resident enzyme acyl coenzyme A: cholesteryl acyl transferase (ACAT) converts free cholesterol to CE and stores it in lipid droplets (Chang et al. 1997). ACAT provides a rapid high capacity mechanism for dealing with cholesterol excess, while the SREBP2 mechanism maintains overall cholesterol homeostasis.

In order for cholesterol levels sensed in the ER to reflect the cholesterol distribution in other organelles, such as the plasma membrane, there must exist mechanisms for rapid redistribution of cholesterol among these organelles. There is relatively little direct vesicular transport of lipids from late endosomes or the plasma membrane to the ER, which suggests the potential importance of non-vesicular transport mechanisms. Despite the known importance of cholesterol homeostasis, there are fundamental gaps in our understanding of how cholesterol moves among organelles.

As diagramed in Figure 2, significant differences in cholesterol concentration are maintained among organelles. In the plasma membrane of mammalian cells, cholesterol is $\sim 35$ mol\% of the lipids (Ikonen 2008). Cholesterol can flip rapidly (Lange et al. 1981; Steck et al. 2002) between the leaflets of the bilayer, and it is enriched in the cytoplasmic leaflet (Mondal et al. 2009; Schroeder et al. 1991). In contrast, cholesterol accounts for only $5 \mathrm{~mol} \%$ of total lipids in the ER (Radhakrishnan et al. 2008). From electron microscopy of filipin-labeled cells (Blanchette-Mackie and Pentchev 1998), it has been suggested that the concentration of cholesterol increases from the cis-Golgi to the trans-Golgi. In the endocytic pathway cholesterol 
is highly enriched in the endocytic recycling compartment (ERC) (Hao et al. 2002). In part, this unequal distribution of cholesterol among cellular membrane compartments can be attributed to the differences in the cholesterol-lipid interactions resulting from the differences in the organelle lipid composition (Iaea and Maxfield 2015; Lange and Steck 2008; Mesmin and Maxfield 2009). The plasma membrane is relatively enriched in lipids that can stabilize cholesterol in the bilayer, while the ER is enriched in unsaturated lipids that provide weak stabilization of cholesterol. Because of these differences in cholesterol stabilization, these membranes can have unequal cholesterol concentrations even if sterol transporters bring them close to chemical equilibrium with one another.

We have studied two aspects of intracellular sterol transport in mammalian cells. In one set of studies, we have used a naturally occurring fluorescent sterol, dehydroergosterol (DHE) to track the movement of sterol in cells. DHE is a reasonably good analog of cholesterol in many of its biophysical properties and is a substrate for enzymes such as ACAT or LAL (Pipalia et al. 2007; Wustner et al. 2005). As discussed below, these studies have led to an appreciation of the importance of a soluble cytoplasmic sterol transport protein, STARD4, in intracellular cholesterol transport. In the second set of studies we have examined the defects in the NPC1 protein that lead to the recessive hereditary disorder Niemann Pick disease type C (NPC). These studies have led to an understanding of the role of ER-associated protein degradation (ERAD) in the loss of mutated NPC1 protein, and they have led to a potential therapeutic approach in which histone deacetylase inhibitors reduce ERAD of the NPC1 protein and allow functional mutants of the NPC1 protein to be delivered to the LE/Ly (Pipalia et.al, 2011; Pragasstis et.al. 2015). 


\section{Intracellular sterol trafficking mechanisms}

Cholesterol can move among organelles by vesicular and non-vesicular transport mechanisms (Iaea and Maxfield 2015; Maxfield and Menon 2006; Maxfield and Wustner 2002). There is substantial evidence for high rates of non-vesicular sterol transport in cells (Liscum and Faust 1989; Maxfield and Mondal 2006). Since cholesterol is very poorly soluble in water and has a high energy barrier for desorption from membranes (Maxfield and Menon 2006), nonvesicular transport requires binding to carrier proteins. Newly synthesized sterols are transported from the ER to the plasma membrane when vesicular trafficking is inhibited by either genetic or pharmacological intervention (Urbani and Simoni 1990). Furthermore, dehydroergosterol (DHE), a naturally fluorescent sterol, equilibrates between the plasma membrane and the ERC within minutes, and this transport is not drastically altered under ATP-depleted conditions that block vesicle transport (Hao et al. 2002; Mesmin et al. 2011). This suggests that the majority of cholesterol transport is mediated by non-vesicular, carrier-mediated transport mechanisms. There are several protein families that are classified as lipid transfer proteins, which have been shown to be capable of transferring lipids between membranes (Beh et al. 2012; Prinz 2007; Soccio and Breslow 2003). Lipid transfer proteins can interact directly with organelles to extract lipid and then diffuse in complex with the lipid to deliver it to acceptor membranes (Iaea et al. 2015). Lipid transfer proteins can significantly increase the rates of lipid transfer between liposomes in vitro (Iaea et al. 2015; Lusa et al. 2003; Mesmin et al. 2011). In the case of membrane contact sites (Levine 2004), where two organelles are held together in close proximity, the distance that the lipid-protein complex must travel is reduced, facilitating rapid exchange of sterol (de Saint-Jean et al. 2011; Mesmin et al. 2013) or other lipids (Chung et al. 2015; Moser von Filseck et al. 2015) between the membranes. 
One gene family of lipid transfer proteins that have been implicated in such trafficking is the steroidogenic acute regulatory protein (StAR)-related lipid-transfer (START) domain family (Clark 2012). There are 15 members of the mammalian START family (Alpy and Tomasetto 2005) that can be classified into six subfamilies based on ligand specificity and domain architecture (Clark 2012). Among the cholesterol specific and soluble START proteins, STARD4 has been shown to increase the extent of cholesteryl ester accumulation in lipid droplets in an ACAT-dependent manner (Garbarino et al. 2012; Rodriguez-Agudo et al. 2011), and its expression is controlled at the transcriptional level by sterols (Soccio et al. 2002). STARD4 has been implicated as an important sterol transport protein involved in maintaining cholesterol homeostasis (Garbarino et al. 2012; Iaea et al. 2014; Mesmin et al. 2011). However, the mechanisms by which soluble lipid transfer proteins, including STARD4, efficiently target to specific membranes to solubilize and extract or deliver sterol remain unclear.

\section{Role of STARD4 in sterol transport}

STARD4 is composed of a single START domain that lacks a subcellular localization motif. As a result, STARD4 is distributed throughout the cytosol (Iaea et al. 2014) but transiently interacts with organelles such as the ER (Rodriguez-Agudo et al. 2011) and ERC (Mesmin et al. 2011). However, the exact mechanism underlying STARD4 recruitment to membranes is only partially understood (Iaea et al. 2015; Mesmin et al. 2011).

Structurally, STARD4 has a surface patch of basic residues and a non-polar $\Omega_{1}$ loop that are separated by the C-terminal $\alpha$-helix (Iaea et al. 2015; Romanowski et al. 2002) (Figure 3). The basic patch of STARD4 interacts with anionic lipids, such as phosphatidylserine (PS). Mutational studies in which lysines in the basic patch were replaced with alanine resulted in attenuated sterol transfer activity (Iaea et al. 2015; Mesmin et al. 2011). This attenuated activity 
is presumably due to perturbed electrostatic interactions between STARD4 and anionic lipid head groups that are required for optimal orientation to the membrane for docking (Iaea et al. 2015).

Following recruitment of STARD4 to anionic membranes, it was suggested from molecular dynamics studies that movement of the non-polar $\Omega_{1}$ loop would be sufficient to allow sterol absorption and release (Murcia et al. 2006). Introduction of a negative charge into the apex of the $\Omega_{1}$ loop resulted in 100-fold reduction in sterol transfer activity (Iaea et al. 2015). However, this reduction in sterol transfer activity was not the result of charge-charge repulsion but rather a stabilization of the entire protein as well as attenuated membrane interaction and activity (Iaea et al. 2015). Additionally, it was found that the C-terminal $\alpha$-helix, and not the $\Omega_{1}$ loop, inserts into the bilayer.

From the current data (Iaea et al. 2015; Kudo et al. 2010; Mesmin et al. 2011; Murcia et al. 2006; Roostaee et al. 2009) it can be envisioned that conformational changes of the $\Omega_{1}$ loop and C-terminal $\alpha$-helix stabilized by interaction with the bilayer would allow enough space and time for the sterol or lipid to diffuse, polar component first, into the ligand binding pocket. Following ligand binding, the protein can disengage from the membrane to transfer the sterol or lipid to another cellular organelle. STARD4 sterol transport activity requires a surface basic patch as well as movement of the $\Omega_{1}$ loop along with rearrangement and insertion of the Cterminal $\alpha$-helix into the membrane (Iaea et al. 2015).

We have limited quantitative information regarding how lipid transfer proteins facilitate sterol transport. In vitro, STARD4 sterol transfer activity is dependent on interaction with anionic lipids such as PS (Iaea et al. 2015; Mesmin et al. 2011; Romanowski et al. 2002). Since PS is abundant in several membrane organelles (Kay and Grinstein 2013; van Meer 2005; van 
Meer et al. 2008), this may facilitate STARD4 recruitment to various organelles. However, it is possible that STARD4 also interacts with other anionic lipids such as phosphatidylinositol phosphates (PIPs), which are heterogeneously enriched on cellular organelles (Hammond and Balla 2015; Maxfield and Hao 2013) (Figure 2). Specific PIPs have been shown to modulate the activity of two sterol transport proteins, Osh4p (de Saint-Jean et al. 2011) and Oxysterol Binding Protein (OSBP) (Mesmin et al. 2013). For both of these lipid transfer proteins, it was found that they exchanged sterol in the ER for phosphatidylinositol-4-phosphate (PI4P) in the Golgi, generating a gradient to enrich sterol at the expense of PI4P (Holthuis and Menon 2014; Levine and Menon 2013). In this way, the enrichment of PI4P on the Golgi drives the vectorial transport of sterol from the ER to the Golgi. This mechanism of vectorial transport of lipid by exchanging with PI4P is not unique for sterol and has been recently described for PS transfer mediated by ORP5/8 (Chung et al. 2015) and Osh6/7 (Moser von Filseck et al. 2015). It remains unknown if the activity of other soluble sterol transport proteins, including STARD4, is tied to distribution of PIPs or other anionic lipids in cells.

Cholesterol is distributed throughout organelles and the membrane bilayer. It has a rapid lateral and transverse mobility and has dramatic effects on the organization of its surrounding membrane lipids and proteins (Pani et al. 2008). There is increasing evidence that the majority of sterol trafficking is carried out by non-vesicular mechanisms. The presence of numerous sterol transport proteins demonstrates that cells can rapidly redistribute sterol among organelles and respond to alterations in cholesterol levels to maintain cellular lipid homeostasis. An important question for the next few years is to elucidate the trafficking kinetics of sterols into and out of organelles and how these transport steps are integrated into the overall cellular homeostasis. 


\section{Aberrant sterol transport in Niemann Pick disease type $\mathbf{C}$}

Niemann Pick disease type C (NPC) is a rare autosomal recessive disease that is associated with abnormal accumulation of unesterified cholesterol, glycosphingolipids, and other lipids in late endosome and lysosomes (LE/Ly) of many cell types. In NPC patients LDL-derived cholesterol fails to exit LE/Ly due to mutation in the NPC1 or NPC2 genes, and this causes lipids to accumulate in lysosome like storage organelles (LSO). $95 \%$ of all NPC disease is due to mutations in the NPC1 gene (Ory 2000). The major pathology associated with NPC disease is in the central nervous system, but abnormal lipid accumulation occurs in peripheral organs, leading to pathology in these tissues as well. The incidence had been estimated to be between 1:120,000 and 1:150,000 live births (Vanier 2010), but recent large scale sequencing data suggest that it may be about three times more prevalent than the previous estimates (Bauer et al. 2013; Wassif et al. 2015). More than 200 different polymorphisms in patients are reported (Fernandez-Valero et al. 2005; Jahnova et al. 2014; Park et al. 2003; Pina-Aguilar et al. 2014; Runz et al. 2008), with the most prevalent mutation, I1061T, accounting for about $15 \%$ of mutant alleles (Rauniyar et al. 2015; Yamamoto et al. 1999).

\section{Therapies for NPC disease}

Currently there is no therapy available for NPC1 disease. One drug approved in the European Union (but not the USA) for the treatment of NPC disease is Zavesca (Miglustat), which inhibits glycosphingolipid synthesis (Patterson et al. 2007). Glycolipids are among the lipids that accumulate in LSOs as a consequence of the elevated cholesterol levels, and reducing their abundance might reduce the effects of lipid storage. Zavesca treatment has been shown to slow the disease progression (Alobaidy 2015; Di Rocco et al. 2012; Skorpen et al. 2012). 
Two types of therapies for NPC disease are currently in small clinical trials. Hydroxypropyl- $\beta$ cyclodextrin (HPBCD) is a cyclic sugar molecule with a hydrophobic core that can solubilize cholesterol. As discussed below, HPBCD can bypass the need for NPC1 and NPC2 in LE/Ly (Abi-Mosleh et al. 2009; Rosenbaum et al. 2010). Another potential therapy was found in a large scale screen for small molecules that would reduce the mutant phenotype in NPC1 mutant fibroblasts. Among the effective molecules, histone deacetylase inhibitors (HDACi) were shown to reduce the accumulation of cholesterol and other lipids found in patient fibroblasts harboring the NPC1 $1^{\mathrm{I} 1061 \mathrm{~T}}$ and other mutations (Pipalia et al. 2011). Some HDACi have been approved by the FDA as cancer therapeutics, and their potential for treatment of NPC1 disease has been reviewed (Helquist et al. 2013).

Cyclodextrins are frequently used as water soluble carriers for hydrophobic drugs (Brewster and Loftsson 2007), and they were used as carriers for steroid hormones as a potential treatment for $n p c l^{-/-}$mice. In two studies (Davidson et al. 2009; Ramirez et al. 2010) the mice treated with HPBCD alone showed delayed neuropathology and extended lifespan compared with untreated $n p c 1^{-/-}$mice. Repeated injections of HPBCD could extend the lifespan of $n p c 1^{-/-}$ mice from 80-120 days to over a year (Ramirez et al. 2010). Cholesterol homeostatic mechanisms were restored in the liver of treated mice. The rate of cholesterol synthesis was reduced to normal levels; CE levels increased; and mRNA levels of SREBP2 target genes - LDL receptor and HMG-CoA reductase were normalized. These changes indicated that HPBCD treatment normalized cholesterol distribution in the liver cells (Lopez et al. 2014). Studies in cell culture models showed that treatment with $100 \mu \mathrm{M}$ HPBCD for 2-3 days could clear the accumulated cholesterol from LSOs and restore normal cholesterol homeostasis (Abi-Mosleh et 
al. 2009; Rosenbaum et al. 2010). These studies also showed that delivery of the cyclodextrins to the LE/Ly be nonselective endocytosis was essential for their beneficial effects.

HPBCD treatment also dramatically slowed the onset of neurological symptoms in a cat model of NPC1 disease (Vite et al. 2015; Ward et al. 2010). In the cat, direct injection of the HPBCD into the CNS was required because of poor penetration through the blood brain barrier (Pontikis et al. 2013; Tortelli et al. 2014). These studies led to a small clinical trial in humans that included delivery to the CNS (Clinical-Trial). This study, which was mainly a safety trial, is being expanded into a larger trial to test efficacy (Cyclodextrin-Efficacy-Trial). In addition to poor transport across the blood brain barrier, the cyclodextrins are rapidly excreted in the urine, and these pharmacokinetic properties present therapeutic challenges.

An unbiased small molecule screen conducted on human skin fibroblasts bearing point mutations in NPC1 identified several HDACi that could reduce cholesterol accumulation in LSOs (Pipalia et al. 2011). The SREBP2 mediated feedback system was restored by HDACi treatment. Thus, biosynthesis of cholesterol was normalized, and LDL receptors were down regulated - stopping the influx of endocytosed cholesterol. The level of the mutant NPC1 protein was increased in the treated human fibroblasts with no apparent increase in mRNA (Pipalia et al. 2011). It had been noted previously that nearly all of the NPC $1^{\mathrm{I} 1061 \mathrm{~T}}$ mutant protein was eliminated by ERAD (Gelsthorpe et al. 2008), and treatments that could overcome ERAD led to partial restoration of function. It appears that treatment of some NPC1 mutant cells with HDACi can similarly overcome the elimination of the proteins by ERAD. Consistent with this proposed mechanism, treatment with HDACi did not correct the cholesterol storage in $\mathrm{NPC}^{-/-}$cells or in cells with an NPC2 truncation mutation (FR Maxfield and NH Pipalia, unpublished).

Interestingly, HDACi treatment of Gaucher disease fibroblasts with a folding deficiency 
in B-glucosidase led to restoration of the enzyme activity in LE/Ly (Lu et al. 2011). Treatment with HDACi also promotes secretion of mutant forms of $\alpha 1$-antitrypsin (Bouchecareilh et al. 2012), suggesting that HDACi treatment might be effective in other disorders of protein folding in the ER. The proteostatic effect of Vorinostat, a US FDA-approved HDACi (Vorinostat-FDAApproval), has been studied in cystic fibrosis. Vorinostat treatment allowed a misfolded mutant (CFTR $\triangle F 508)$ to avoid ERAD and be delivered to the-plasma membrane (Hutt et al. 2010; Sondo et al. 2011).

Vorinostat is currently being evaluated in a small safety trial in adult NPC1 patients (Vorinostat-NPC1-Trail). There are several histone deacetylases in humans, and Vorinostat is a broad spectrum HDACi. Based on current evidence, it is likely that inhibitors of HDAC1, 2, or 3 will be most effective (Helquist et al. 2013), but further work is required to determine the optimal specificity. Several pharmaceutical companies are testing more selective HDACi, and it is possible that more selective inhibitors will be effective in treating NPC1 disease with fewer side effects. Vorinostat also has relatively poor brain penetration, and several HDACi (e.g., CI994) (Schroeder et al. 2013) have better brain penetration. There are ongoing efforts to identify optimal HDACi for treatment of NPC1 disease. The recent development of a mouse model with a knock-in of the NPC1 ${ }^{\text {I1061T }}$ mutation (Praggastis et al. 2015) will help in preclinical testing of HDACi. The finding that overcoming ERAD can rescue several NPC1 mutations also leads to searches for other proteostasis regulators as possible therapies.

Acknowledgement: Work in the authors' laboratory has been supported by NIH grant R37DK27083 and by grants from the Ara Parseghian Medical Research Foundation. DBI was supported by NIH grant F31-DK104631. 
Figure 1: Schematic of intracellular trafficking. Cholesterol is synthesized in the endoplasmic reticulum (ER). When cells are depleted of sterols SREBP2-SCAP complex migrates to Golgi where SREBP2 undergoes proteolytic cleavage. The mature form of SREBP2 then translocates to the nucleus where it activates sterol response element (SRE) responsible for upregulating genes involved in cholesterol uptake and synthesis. Cells also obtain cholesterol by receptor mediated endocytosis of low density lipoproteins (LDL), which are delivered to late endosomes (LE) or lysosomes (Ly) where the cholesteryl esters in LDL are hydrolyzed by lysosomal acid lipase (LAL). Free cholesterol is distributed among various organelles by a combination of vesicular and non-vesicular pathways. Excess cholesterol is esterified in the ER by acyl CoA:cholesterol acyl transferase (ACAT) and stored in lipid droplets. In NPC mutant cells free cholesterol is unable to egress from LE/Ly, resulting in accumulation, which forms lysosome like storage organelles (LSO).

Figure 2: Heterogeneous distribution of cellular cholesterol and phosphatidylinositol phosphates. The cholesterol content of membranes varies among cellular organelles. Membrane cholesterol levels are displayed as a heat map with membranes enriched in cholesterol labeled red. Membrane compartments with relatively low cholesterol levels are labeled blue. The inset describes the transbilayer distribution of cholesterol at the plasma membrane (Lange et al. 1981; Mesmin and Maxfield 2009). Phosphoinositides serve as organelle identity molecules (Balla 2013; Di Paolo and De Camilli 2006): PI3P (green), phosphatidylinositol-3- phosphate; PI4P 
(red), phosphatidylinositol-4-phosphate; PI5P (purple), phosphatidylinositol-5-phosphate;

$\mathrm{PI}(3,5) \mathrm{P}_{2}$ (lavender), phosphatidylinositol-(3,5)-bisphosphate; $\mathrm{PI}(3,4) \mathrm{P}_{2}$ (gold),

phosphatidylinositol-(3,4)-bisphosphate; $\mathrm{PI}(4,5) \mathrm{P}_{2}$ (pink), phosphatidylinositol-(4,5)-

bisphosphate; and $\mathrm{PI}(3,4,5) \mathrm{P}_{3}$ (blue), phosphatidylinositol-(3,4,5)-trisphosphate.

Figure 3: Regions of STARD4 involved in membrane interaction and sterol transport. Surface representation of STARD4 colored according to the electrostatic potential (Iaea et al. 2015). The basic patch, C-terminal $\alpha$-helix, Omega-1 $\left(\Omega_{1}\right)$ loop and sterol binding site are identified in the structures. Right, the structure is rotated $90^{\circ}$ about the $\mathrm{z}$-axis for a view of the sterol binding pocket of STARD4.

\section{$\underline{\text { References }}$}

Abi-Mosleh, L., Infante, R.E., Radhakrishnan, A., Goldstein, J.L., and Brown, M.S. 2009. Cyclodextrin overcomes deficient lysosome-to-endoplasmic reticulum transport of cholesterol in Niemann-Pick type C cells. Proc Natl Acad Sci U S A 106(46): 19316-19321. doi: 0910916106 [pii]

10.1073/pnas.0910916106.

Alobaidy, H. 2015. Recent advances in the diagnosis and treatment of niemann-pick disease type $\mathrm{C}$ in children: a guide to early diagnosis for the general pediatrician. Int J Pediatr 2015: 816593. doi: 10.1155/2015/816593.

Alpy, F., and Tomasetto, C. 2005. Give lipids a START: the StAR-related lipid transfer (START) domain in mammals. J Cell Sci 118(Pt 13): 2791-2801. doi: 118/13/2791 [pii]

10.1242/jcs.02485.

Balla, T. 2013. Phosphoinositides: tiny lipids with giant impact on cell regulation. Physiol Rev 93(3): 1019-1137. doi: 10.1152/physrev.00028.2012.

Bauer, P., Balding, D.J., Klunemann, H.H., Linden, D.E., Ory, D.S., Pineda, M., Priller, J., Sedel, F., Muller, A., Chadha-Boreham, H., Welford, R.W., Strasser, D.S., and Patterson, M.C. 2013. Genetic screening for Niemann-Pick disease type $\mathrm{C}$ in adults with neurological and psychiatric symptoms: findings from the ZOOM study. Hum Mol Genet 22(21): 4349-4356. doi: 10.1093/hmg/ddt284.

Beh, C.T., McMaster, C.R., Kozminski, K.G., and Menon, A.K. 2012. A detour for yeast oxysterol binding proteins. The Journal of biological chemistry 287(14): 11481-11488. doi: 10.1074/jbc.R111.338400. 
Blanchette-Mackie, E.J., and Pentchev, P.G. 1998. Cholesterol distribution in Golgi, lysosomes, and endoplasmic reticulum. In Intracellular Cholesterol Trafficking: 53-74.

Bouchecareilh, M., Hutt, D.M., Szajner, P., Flotte, T.R., and Balch, W.E. 2012. Histone deacetylase inhibitor (HDACi) suberoylanilide hydroxamic acid (SAHA)-mediated correction of alpha1-antitrypsin deficiency. The Journal of biological chemistry 287(45): 38265-38278. doi: 10.1074/jbc.M112.404707.

Brewster, M.E., and Loftsson, T. 2007. Cyclodextrins as pharmaceutical solubilizers. Adv Drug Deliv Rev 59(7): 645-666. doi: 10.1016/j.addr.2007.05.012.

Brown, M.S., Anderson, R.G., and Goldstein, J.L. 1983. Recycling receptors: the round-trip itinerary of migrant membrane proteins. Cell 32(3): 663-667.

Brown, M.S., Dana, S.E., and Goldstein, J.L. 1975. Receptor-dependent hydrolysis of cholesteryl esters contained in plasma low density lipoprotein. Proc Natl Acad Sci U S A 72(8): 2925-2929. Brown, M.S., and Goldstein, J.L. 1997. The SREBP pathway: regulation of cholesterol metabolism by proteolysis of a membrane-bound transcription factor. Cell 89(3): 331-340. Chang, T.Y., Chang, C.C., and Cheng, D. 1997. Acyl-coenzyme A:cholesterol acyltransferase. Annu Rev Biochem 66: 613-638. doi: 10.1146/annurev.biochem.66.1.613.

Chung, J., Torta, F., Masai, K., Lucast, L., Czapla, H., Tanner, L.B., Narayanaswamy, P., Wenk, M.R., Nakatsu, F., and De Camilli, P. 2015. INTRACELLULAR TRANSPORT.

PI4P/phosphatidylserine countertransport at ORP5- and ORP8-mediated ER-plasma membrane contacts. Science 349(6246): 428-432. doi: 10.1126/science.aab1370.

Clark, B.J. 2012. The mammalian START domain protein family in lipid transport in health and disease. The Journal of endocrinology 212(3): 257-275. doi: 10.1530/JOE-11-0313.

Clinical-Trial. https://clinicaltrials.gov/ct2/show/NCT01747135.

Cyclodextrin-Efficacy-Trial. https://clinicaltrials.gov/ct2/show/NCT02534844.

Davidson, C.D., Ali, N.F., Micsenyi, M.C., Stephney, G., Renault, S., Dobrenis, K., Ory, D.S., Vanier, M.T., and Walkley, S.U. 2009. Chronic cyclodextrin treatment of murine Niemann-Pick $\mathrm{C}$ disease ameliorates neuronal cholesterol and glycosphingolipid storage and disease progression. PLoS One 4(9): e6951. doi: 10.1371/journal.pone.0006951.

de Saint-Jean, M., Delfosse, V., Douguet, D., Chicanne, G., Payrastre, B., Bourguet, W., Antonny, B., and Drin, G. 2011. Osh4p exchanges sterols for phosphatidylinositol 4-phosphate between lipid bilayers. J Cell Biol 195(6): 965-978. doi: 10.1083/jcb.201104062.

Di Paolo, G., and De Camilli, P. 2006. Phosphoinositides in cell regulation and membrane dynamics. Nature 443(7112): 651-657. doi: 10.1038/nature05185.

Di Rocco, M., Dardis, A., Madeo, A., Barone, R., and Fiumara, A. 2012. Early miglustat therapy in infantile Niemann-Pick disease type C. Pediatr Neurol 47(1): 40-43. doi:

10.1016/j.pediatrneurol.2012.04.005.

Dunn, K.W., and Maxfield, F.R. 1992. Delivery of ligands from sorting endosomes to late endosomes occurs by maturation of sorting endosomes. J Cell Biol 117(2): 301-310.

Fernandez-Valero, E.M., Ballart, A., Iturriaga, C., Lluch, M., Macias, J., Vanier, M.T., Pineda, M., and Coll, M.J. 2005. Identification of 25 new mutations in 40 unrelated Spanish NiemannPick type C patients: genotype-phenotype correlations. Clin Genet 68(3): 245-254. doi: 10.1111/j.1399-0004.2005.00490.x.

Friedland, N., Liou, H.L., Lobel, P., and Stock, A.M. 2003. Structure of a cholesterol-binding protein deficient in Niemann-Pick type C2 disease. Proc Natl Acad Sci U S A 100(5): 25122517. doi: 10.1073/pnas.0437840100. 
Garbarino, J., Pan, M., Chin, H.F., Lund, F.W., Maxfield, F.R., and Breslow, J.L. 2012.

STARD4 knockdown in HepG2 cells disrupts cholesterol trafficking associated with the plasma membrane, ER, and ERC. Journal of lipid research 53(12): 2716-2725. doi:

10.1194/jlr.M032227.

Gelsthorpe, M.E., Baumann, N., Millard, E., Gale, S.E., Langmade, S.J., Schaffer, J.E., and Ory, D.S. 2008. Niemann-Pick type C1 I1061T mutant encodes a functional protein that is selected for endoplasmic reticulum-associated degradation due to protein misfolding. The Journal of biological chemistry 283(13): 8229-8236. doi: 10.1074/jbc.M708735200.

Ghosh, R.N., Gelman, D.L., and Maxfield, F.R. 1994. Quantification of low density lipoprotein and transferrin endocytic sorting HEp2 cells using confocal microscopy. J Cell Sci 107 ( Pt 8): 2177-2189.

Goldstein, J.L., and Brown, M.S. 2001. Molecular medicine. The cholesterol quartet. Science 292(5520): 1310-1312.

Hammond, G.R., and Balla, T. 2015. Polyphosphoinositide binding domains: Key to inositol lipid biology. Biochim Biophys Acta 1851(6): 746-758. doi: 10.1016/j.bbalip.2015.02.013. Hao, M., Lin, S.X., Karylowski, O.J., Wustner, D., McGraw, T.E., and Maxfield, F.R. 2002. Vesicular and non-vesicular sterol transport in living cells. The endocytic recycling compartment is a major sterol storage organelle. The Journal of biological chemistry 277(1): 609-617. doi: 10.1074/jbc.M108861200.

Helquist, P., Maxfield, F.R., Wiech, N.L., and Wiest, O. 2013. Treatment of Niemann--pick type $\mathrm{C}$ disease by histone deacetylase inhibitors. Neurotherapeutics 10(4): 688-697. doi: 10.1007/s13311-013-0217-2.

Holthuis, J.C., and Menon, A.K. 2014. Lipid landscapes and pipelines in membrane homeostasis. Nature 510(7503): 48-57. doi: 10.1038/nature13474.

Hutt, D.M., Herman, D., Rodrigues, A.P., Noel, S., Pilewski, J.M., Matteson, J., Hoch, B., Kellner, W., Kelly, J.W., Schmidt, A., Thomas, P.J., Matsumura, Y., Skach, W.R., Gentzsch, M., Riordan, J.R., Sorscher, E.J., Okiyoneda, T., Yates, J.R., 3rd, Lukacs, G.L., Frizzell, R.A., Manning, G., Gottesfeld, J.M., and Balch, W.E. 2010. Reduced histone deacetylase 7 activity restores function to misfolded CFTR in cystic fibrosis. Nat Chem Biol 6(1): 25-33. doi: 10.1038/nchembio.275.

Iaea, D.B., Dikiy, I., Kiburu, I., Eliezer, D., and Maxfield, F.R. 2015. STARD4 Membrane Interactions and Sterol Binding. Biochemistry 54(30): 4623-4636. doi:

10.1021/acs.biochem.5b00618.

Iaea, D.B., Mao, S., and Maxfield, F.R. 2014. Steroidogenic Acute Regulatory Protein-related Lipid Transfer (START) Proteins in Non-vesicular Cholesterol Transport. In Cholesterol Transporters of the START Domain Protein Family in Health and Disease. Edited by B.J. Clark and D.M. Stocco. Springer New York. pp. 173-188.

Iaea, D.B., and Maxfield, F.R. 2015. Cholesterol trafficking and distribution. Essays in biochemistry 57: 43-55. doi: 10.1042/bse0570043.

Ikonen, E. 2008. Cellular cholesterol trafficking and compartmentalization. Nat Rev Mol Cell Biol 9(2): 125-138. doi: nrm2336 [pii]

10.1038/nrm2336.

Infante, R.E., Wang, M.L., Radhakrishnan, A., Kwon, H.J., Brown, M.S., and Goldstein, J.L. 2008. NPC2 facilitates bidirectional transfer of cholesterol between NPC1 and lipid bilayers, a step in cholesterol egress from lysosomes. Proc Natl Acad Sci U S A 105(40): 15287-15292. doi: $10.1073 /$ pnas.0807328105. 
Jahnova, H., Dvorakova, L., Vlaskova, H., Hulkova, H., Poupetova, H., Hrebicek, M., and Jesina, P. 2014. Observational, retrospective study of a large cohort of patients with NiemannPick disease type $\mathrm{C}$ in the Czech Republic: a surprisingly stable diagnostic rate spanning almost 40 years. Orphanet J Rare Dis 9: 140. doi: 10.1186/s13023-014-0140-6.

Kay, J.G., and Grinstein, S. 2013. Phosphatidylserine-mediated cellular signaling. Adv Exp Med Biol 991: 177-193. doi: 10.1007/978-94-007-6331-9_10.

Kudo, N., Kumagai, K., Matsubara, R., Kobayashi, S., Hanada, K., Wakatsuki, S., and Kato, R. 2010. Crystal structures of the CERT START domain with inhibitors provide insights into the mechanism of ceramide transfer. Journal of molecular biology 396(2): 245-251. doi: 10.1016/j.jmb.2009.12.029.

Kwon, H.J., Abi-Mosleh, L., Wang, M.L., Deisenhofer, J., Goldstein, J.L., Brown, M.S., and Infante, R.E. 2009. Structure of N-terminal domain of NPC1 reveals distinct subdomains for binding and transfer of cholesterol. Cell 137(7): 1213-1224. doi: 10.1016/j.cell.2009.03.049. Lange, Y., Dolde, J., and Steck, T.L. 1981. The rate of transmembrane movement of cholesterol in the human erythrocyte. The Journal of biological chemistry 256(11): 5321-5323.

Lange, Y., and Steck, T.L. 2008. Cholesterol homeostasis and the escape tendency (activity) of plasma membrane cholesterol. Progress in lipid research 47(5): 319-332. doi:

10.1016/j.plipres.2008.03.001.

Levine, T. 2004. Short-range intracellular trafficking of small molecules across endoplasmic reticulum junctions. Trends in cell biology 14(9): 483-490. doi: 10.1016/j.tcb.2004.07.017. Levine, T.P., and Menon, A.K. 2013. A protein pair with PIPs inside. Structure 21(7): 10701071. doi: 10.1016/j.str.2013.06.010.

Liscum, L., and Faust, J.R. 1989. The intracellular transport of low density lipoprotein-derived cholesterol is inhibited in Chinese hamster ovary cells cultured with 3-beta-[2-

(diethylamino)ethoxy] androst-5-en-17-one. The Journal of biological chemistry 264(20): 1179611806.

Lopez, A.M., Terpack, S.J., Posey, K.S., Liu, B., Ramirez, C.M., and Turley, S.D. 2014. Systemic administration of 2-hydroxypropyl-beta-cyclodextrin to symptomatic Npc1-deficient mice slows cholesterol sequestration in the major organs and improves liver function. Clin Exp Pharmacol Physiol 41(10): 780-787. doi: 10.1111/1440-1681.12285.

Lu, J., Yang, C., Chen, M., Ye, D.Y., Lonser, R.R., Brady, R.O., and Zhuang, Z. 2011. Histone deacetylase inhibitors prevent the degradation and restore the activity of glucocerebrosidase in Gaucher disease. Proc Natl Acad Sci U S A 108(52): 21200-21205. doi:

10.1073/pnas.1119181109.

Lusa, S., Heino, S., and Ikonen, E. 2003. Differential mobilization of newly synthesized cholesterol and biosynthetic sterol precursors from cells. The Journal of biological chemistry 278(22): 19844-19851. doi: 10.1074/jbc.M212503200.

Maxfield, F., and Hao, M. 2013. Lipid Trafficking in Cells. In Encyclopedia of Biophysics.

Edited by G.K. Roberts. Springer Berlin Heidelberg. pp. 1289-1296.

Maxfield, F.R., and Menon, A.K. 2006. Intracellular sterol transport and distribution. Curr Opin Cell Biol 18(4): 379-385. doi: 10.1016/j.ceb.2006.06.012.

Maxfield, F.R., and Mondal, M. 2006. Sterol and lipid trafficking in mammalian cells. Biochem Soc Trans 34(Pt 3): 335-339. doi: 10.1042/BST0340335.

Maxfield, F.R., and Wustner, D. 2002. Intracellular cholesterol transport. The Journal of clinical investigation 110(7): 891-898. doi: 10.1172/JCI16500. 
Mesmin, B., Bigay, J., Moser von Filseck, J., Lacas-Gervais, S., Drin, G., and Antonny, B. 2013. A four-step cycle driven by $\mathrm{PI}(4) \mathrm{P}$ hydrolysis directs sterol/PI(4)P exchange by the ER-Golgi tether OSBP. Cell 155(4): 830-843. doi: 10.1016/j.cell.2013.09.056.

Mesmin, B., and Maxfield, F.R. 2009. Intracellular sterol dynamics. Biochim Biophys Acta 1791(7): 636-645. doi: S1388-1981(09)00073-0 [pii]

10.1016/j.bbalip.2009.03.002.

Mesmin, B., Pipalia, N.H., Lund, F.W., Ramlall, T.F., Sokolov, A., Eliezer, D., and Maxfield, F.R. 2011. STARD4 abundance regulates sterol transport and sensing. Molecular Biology of the Cell 22(21): 4004-4015. doi: DOI 10.1091/mbc.E11-04-0372.

Mondal, M., Mesmin, B., Mukherjee, S., and Maxfield, F.R. 2009. Sterols are mainly in the cytoplasmic leaflet of the plasma membrane and the endocytic recycling compartment in $\mathrm{CHO}$ cells. Mol Biol Cell 20(2): 581-588. doi: 10.1091/mbc.E08-07-0785.

Moser von Filseck, J., Copic, A., Delfosse, V., Vanni, S., Jackson, C.L., Bourguet, W., and Drin, G. 2015. INTRACELLULAR TRANSPORT. Phosphatidylserine transport by ORP/Osh proteins is driven by phosphatidylinositol 4-phosphate. Science 349(6246): 432-436. doi:

10.1126/science.aab1346.

Murcia, M., Faraldo-Gomez, J.D., Maxfield, F.R., and Roux, B. 2006. Modeling the structure of the StART domains of MLN64 and StAR proteins in complex with cholesterol. Journal of lipid research 47(12): 2614-2630. doi: M600232-JLR200 [pii]

10.1194/jlr.M600232-JLR200.

Ory, D.S. 2000. Niemann-Pick type C: A disorder of cellular cholesterol trafficking. Biochimica et Biophysica Acta (BBA) - Molecular and Cell Biology of Lipids 1529(1-3): 331-339. doi: http://dx.doi.org/10.1016/S1388-1981(00)00158-X.

Pani, B., Ong, H.L., Liu, X., Rauser, K., Ambudkar, I.S., and Singh, B.B. 2008. Lipid rafts determine clustering of STIM1 in endoplasmic reticulum-plasma membrane junctions and regulation of store-operated Ca2+ entry (SOCE). The Journal of biological chemistry 283(25): 17333-17340. doi: 10.1074/jbc.M800107200.

Park, W.D., O'Brien, J.F., Lundquist, P.A., Kraft, D.L., Vockley, C.W., Karnes, P.S., Patterson, M.C., and Snow, K. 2003. Identification of 58 novel mutations in Niemann-Pick disease type C: correlation with biochemical phenotype and importance of PTC1-like domains in NPC1. Hum Mutat 22(4): 313-325. doi: 10.1002/humu.10255.

Patterson, M.C., Vecchio, D., Prady, H., Abel, L., and Wraith, J.E. 2007. Miglustat for treatment of Niemann-Pick C disease: a randomised controlled study. Lancet Neurol 6(9): 765-772. doi:

S1474-4422(07)70194-1 [pii]

10.1016/S1474-4422(07)70194-1.

Pina-Aguilar, R.E., Vera-Loaiza, A., Chacon-Camacho, O.F., Zenteno, J.C., Nunez-Orozco, L., and Santillan-Hernandez, Y. 2014. Clinical and genetic characteristics of mexican patients with juvenile presentation of niemann-pick type C disease. Case Rep Neurol Med 2014: 785890. doi: $10.1155 / 2014 / 785890$.

Pipalia, N.H., Cosner, C.C., Huang, A., Chatterjee, A., Bourbon, P., Farley, N., Helquist, P., Wiest, O., and Maxfield, F.R. 2011. Histone deacetylase inhibitor treatment dramatically reduces cholesterol accumulation in Niemann-Pick type $\mathrm{C} 1$ mutant human fibroblasts. Proc Natl Acad Sci U S A 108(14): 5620-5625. doi: 10.1073/pnas.1014890108. 
Pipalia, N.H., Hao, M., Mukherjee, S., and Maxfield, F.R. 2007. Sterol, protein and lipid trafficking in Chinese hamster ovary cells with Niemann-Pick type C1 defect. Traffic 8(2): 130141. doi: 10.1111/j.1600-0854.2006.00513.x.

Pontikis, C.C., Davidson, C.D., Walkley, S.U., Platt, F.M., and Begley, D.J. 2013. Cyclodextrin alleviates neuronal storage of cholesterol in Niemann-Pick $\mathrm{C}$ disease without evidence of detectable blood-brain barrier permeability. J Inherit Metab Dis 36(3): 491-498. doi: 10.1007/s10545-012-9583-x.

Praggastis, M., Tortelli, B., Zhang, J., Fujiwara, H., Sidhu, R., Chacko, A., Chen, Z., Chung, C., Lieberman, A.P., Sikora, J., Davidson, C., Walkley, S.U., Pipalia, N.H., Maxfield, F.R., Schaffer, J.E., and Ory, D.S. 2015. A murine Niemann-Pick C1 I1061T knock-in model recapitulates the pathological features of the most prevalent human disease allele. J Neurosci 35(21): 8091-8106. doi: 10.1523/JNEUROSCI.4173-14.2015.

Prinz, W.A. 2007. Non-vesicular sterol transport in cells. Progress in lipid research 46(6): 297314. doi: DOI 10.1016/j.plipres.2007.06.002.

Radhakrishnan, A., Goldstein, J.L., McDonald, J.G., and Brown, M.S. 2008. Switch-like control of SREBP-2 transport triggered by small changes in ER cholesterol: a delicate balance. Cell Metab 8(6): 512-521. doi: S1550-4131(08)00326-4 [pii]

10.1016/j.cmet.2008.10.008.

Ramirez, C.M., Liu, B., Taylor, A.M., Repa, J.J., Burns, D.K., Weinberg, A.G., Turley, S.D., and Dietschy, J.M. 2010. Weekly cyclodextrin administration normalizes cholesterol metabolism in nearly every organ of the Niemann-Pick type $\mathrm{C} 1$ mouse and markedly prolongs life. Pediatr Res 68(4): 309-315. doi: 10.1203/00006450-201011001-00604

10.1203/PDR.0b013e3181ee4dd2.

Rauniyar, N., Subramanian, K., Lavallee-Adam, M., Martinez-Bartolome, S., Balch, W.E., and Yates, J.R., 3rd. 2015. Quantitative Proteomics of Human Fibroblasts with I1061T Mutation in Niemann-Pick C1 (NPC1) Protein Provides Insights into the Disease Pathogenesis. Mol Cell Proteomics 14(7): 1734-1749. doi: 10.1074/mcp.M114.045609.

Rodriguez-Agudo, D., Calderon-Dominguez, M., Ren, S., Marques, D., Redford, K., MedinaTorres, M.A., Hylemon, P., Gil, G., and Pandak, W.M. 2011. Subcellular localization and regulation of StarD4 protein in macrophages and fibroblasts. Biochim Biophys Acta 1811(10): 597-606. doi: S1388-1981(11)00120-X [pii]

10.1016/j.bbalip.2011.06.028.

Romanowski, M.J., Soccio, R.E., Breslow, J.L., and Burley, S.K. 2002. Crystal structure of the Mus musculus cholesterol-regulated START protein 4 (StarD4) containing a StAR-related lipid transfer domain. Proc Natl Acad Sci U S A 99(10): 6949-6954. doi: 10.1073/pnas.052140699

99/10/6949 [pii].

Roostaee, A., Barbar, E., Lavigne, P., and LeHoux, J.G. 2009. The mechanism of specific binding of free cholesterol by the steroidogenic acute regulatory protein: evidence for a role of the C-terminal alpha-helix in the gating of the binding site. Bioscience reports 29(2): 89-101. doi: 10.1042/BSR20080111.

Rosenbaum, A.I., Zhang, G., Warren, J.D., and Maxfield, F.R. 2010. Endocytosis of betacyclodextrins is responsible for cholesterol reduction in Niemann-Pick type C mutant cells. Proc Natl Acad Sci U S A 107(12): 5477-5482. doi: 0914309107 [pii]

10.1073/pnas.0914309107. 
Runz, H., Dolle, D., Schlitter, A.M., and Zschocke, J. 2008. NPC-db, a Niemann-Pick type C disease gene variation database. Hum Mutat 29(3): 345-350. doi: 10.1002/humu.20636.

Schroeder, F., Nemecz, G., Wood, W.G., Joiner, C., Morrot, G., Ayraut-Jarrier, M., and Devaux, P.F. 1991. Transmembrane distribution of sterol in the human erythrocyte. Biochim Biophys Acta 1066(2): 183-192.

Schroeder, F.A., Chonde, D.B., Riley, M.M., Moseley, C.K., Granda, M.L., Wilson, C.M., Wagner, F.F., Zhang, Y.L., Gale, J., Holson, E.B., Haggarty, S.J., and Hooker, J.M. 2013. FDGPET imaging reveals local brain glucose utilization is altered by class I histone deacetylase inhibitors. Neurosci Lett 550: 119-124. doi: 10.1016/j.neulet.2013.06.016.

Scott, C., and Ioannou, Y.A. 2004. The NPC1 protein: structure implies function. Biochim Biophys Acta 1685(1-3): 8-13. doi: 10.1016/j.bbalip.2004.08.006.

Skorpen, J., Helland, I.B., and Tennoe, B. 2012. Use of miglustat in a child with late-infantileonset Niemann-Pick disease type C and frequent seizures: a case report. J Med Case Rep 6: 383. doi: 10.1186/1752-1947-6-383.

Soccio, R.E., Adams, R.M., Romanowski, M.J., Sehayek, E., Burley, S.K., and Breslow, J.L. 2002. The cholesterol-regulated StarD4 gene encodes a StAR-related lipid transfer protein with two closely related homologues, StarD5 and StarD6. Proc Natl Acad Sci U S A 99(10): 69436948. doi: 10.1073/pnas.052143799

99/10/6943 [pii].

Soccio, R.E., and Breslow, J.L. 2003. StAR-related lipid transfer (START) proteins: mediators of intracellular lipid metabolism. The Journal of biological chemistry 278(25): 22183-22186. doi: 10.1074/jbc.R300003200.

Sondo, E., Tomati, V., Caci, E., Esposito, A.I., Pfeffer, U., Pedemonte, N., and Galietta, L.J. 2011. Rescue of the mutant CFTR chloride channel by pharmacological correctors and low temperature analyzed by gene expression profiling. Am J Physiol Cell Physiol 301(4): C872-885. doi: 10.1152/ajpcell.00507.2010.

Steck, T.L., Ye, J., and Lange, Y. 2002. Probing red cell membrane cholesterol movement with cyclodextrin. Biophysical journal 83(4): 2118-2125. doi: 10.1016/S0006-3495(02)73972-6.

Tortelli, B., Fujiwara, H., Bagel, J.H., Zhang, J., Sidhu, R., Jiang, X., Yanjanin, N.M., Shankar, R.K., Carillo-Carasco, N., Heiss, J., Ottinger, E., Porter, F.D., Schaffer, J.E., Vite, C.H., and Ory, D.S. 2014. Cholesterol homeostatic responses provide biomarkers for monitoring treatment for the neurodegenerative disease Niemann-Pick C1 (NPC1). Hum Mol Genet 23(22): 6022-6033. doi: $10.1093 / \mathrm{hmg} / \mathrm{ddu} 331$.

Urbani, L., and Simoni, R.D. 1990. Cholesterol and vesicular stomatitis virus G protein take separate routes from the endoplasmic reticulum to the plasma membrane. The Journal of biological chemistry 265(4): 1919-1923.

Van Berkel, T.J., Kruijt, J.K., and Koster, J.F. 1975. Identity and activities of lysosomal enzymes in parenchymal and non-parenchymal cells from rat liver. Eur J Biochem 58(1): 145-152.

van Meer, G. 2005. Cellular lipidomics. EMBO J 24(18): 3159-3165. doi:

10.1038/sj.emboj.7600798.

van Meer, G., Voelker, D.R., and Feigenson, G.W. 2008. Membrane lipids: where they are and how they behave. Nat Rev Mol Cell Biol 9(2): 112-124. doi: 10.1038/nrm2330.

Vanier, M.T. 2010. Niemann-Pick disease type C. Orphanet J Rare Dis 5: 16. doi: 10.1186/17501172-5-16.

Vite, C.H., Bagel, J.H., Swain, G.P., Prociuk, M., Sikora, T.U., Stein, V.M., O'Donnell, P., Ruane, T., Ward, S., Crooks, A., Li, S., Mauldin, E., Stellar, S., De Meulder, M., Kao, M.L., 
Ory, D.S., Davidson, C., Vanier, M.T., and Walkley, S.U. 2015. Intracisternal cyclodextrin prevents cerebellar dysfunction and Purkinje cell death in feline Niemann-Pick type $\mathrm{C} 1$ disease. Sci Transl Med 7(276): 276ra226. doi: 10.1126/scitranslmed.3010101. Vorinostat-FDA-Approval. http://www.accessdata.fda.gov/drugsatfda_docs/nda/2006/021991s000_ZolinzaTOC.cfm. Vorinostat-NPC1-Trail. https://clinicaltrials.gov/ct2/show/NCT02124083. Ward, S., O'Donnell, P., Fernandez, S., and Vite, C.H. 2010. 2-hydroxypropyl-beta-cyclodextrin raises hearing threshold in normal cats and in cats with Niemann-Pick type $C$ disease. Pediatr Res 68(1): 52-56. doi: 10.1203/00006450-201011001-00099

10.1203/PDR.0b013e3181df4623.

Wassif, C.A., Cross, J.L., Iben, J., Sanchez-Pulido, L., Cougnoux, A., Platt, F.M., Ory, D.S., Ponting, C.P., Bailey-Wilson, J.E., Biesecker, L.G., and Porter, F.D. 2015. High incidence of unrecognized visceral/neurological late-onset Niemann-Pick disease, type C1, predicted by analysis of massively parallel sequencing data sets. Genet Med. doi: 10.1038/gim.2015.25. Wustner, D., Mondal, M., Tabas, I., and Maxfield, F.R. 2005. Direct observation of rapid internalization and intracellular transport of sterol by macrophage foam cells. Traffic 6(5): 396412. doi: 10.1111/j.1600-0854.2005.00285.x.

Yamamoto, T., Nanba, E., Ninomiya, H., Higaki, K., Taniguchi, M., Zhang, H., Akaboshi, S., Watanabe, Y., Takeshima, T., Inui, K., Okada, S., Tanaka, A., Sakuragawa, N., Millat, G., Vanier, M.T., Morris, J.A., Pentchev, P.G., and Ohno, K. 1999. NPC1 gene mutations in Japanese patients with Niemann-Pick disease type C. Hum Genet 105(1-2): 10-16.

Yamashiro, D.J., and Maxfield, F.R. 1984. Acidification of endocytic compartments and the intracellular pathways of ligands and receptors. J Cell Biochem 26(4): 231-246. doi: $10.1002 /$ jcb. 240260404. 


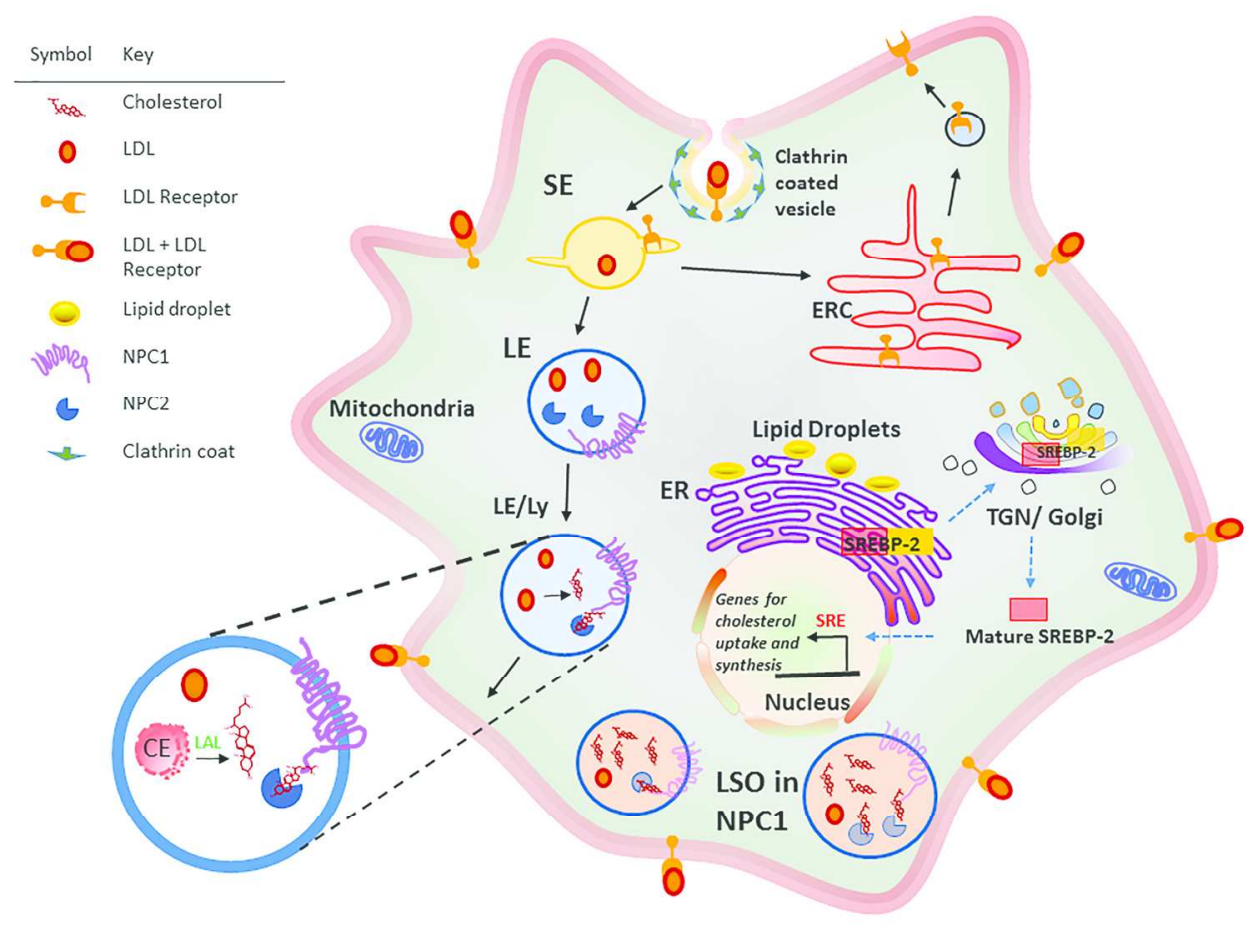

Figure 1: Schematic of intracellular trafficking. Cholesterol is synthesized in the endoplasmic reticulum (ER). When cells are depleted of sterols SREBP2-SCAP complex migrates to Golgi where SREBP2 undergoes proteolytic cleavage. The mature form of SREBP2 then translocates to the nucleus where it activates sterol response element (SRE) responsible for upregulating genes involved in cholesterol uptake and synthesis. Cells also obtain cholesterol by receptor mediated endocytosis of low density lipoproteins (LDL), which are delivered to late endosomes (LE) or lysosomes (Ly) where the cholesteryl esters in LDL are hydrolyzed by lysosomal acid lipase (LAL). Free cholesterol is distributed among various organelles by a combination of vesicular and non-vesicular pathways. Excess cholesterol is esterified in the ER by acyl CoA:cholesterol acyl transferase (ACAT) and stored in lipid droplets. In NPC mutant cells free cholesterol is unable to egress from LE/Ly, resulting in accumulation, which forms lysosome like storage organelles (LSO). $165 \times 123 \mathrm{~mm}(300 \times 300 \mathrm{DPI})$ 


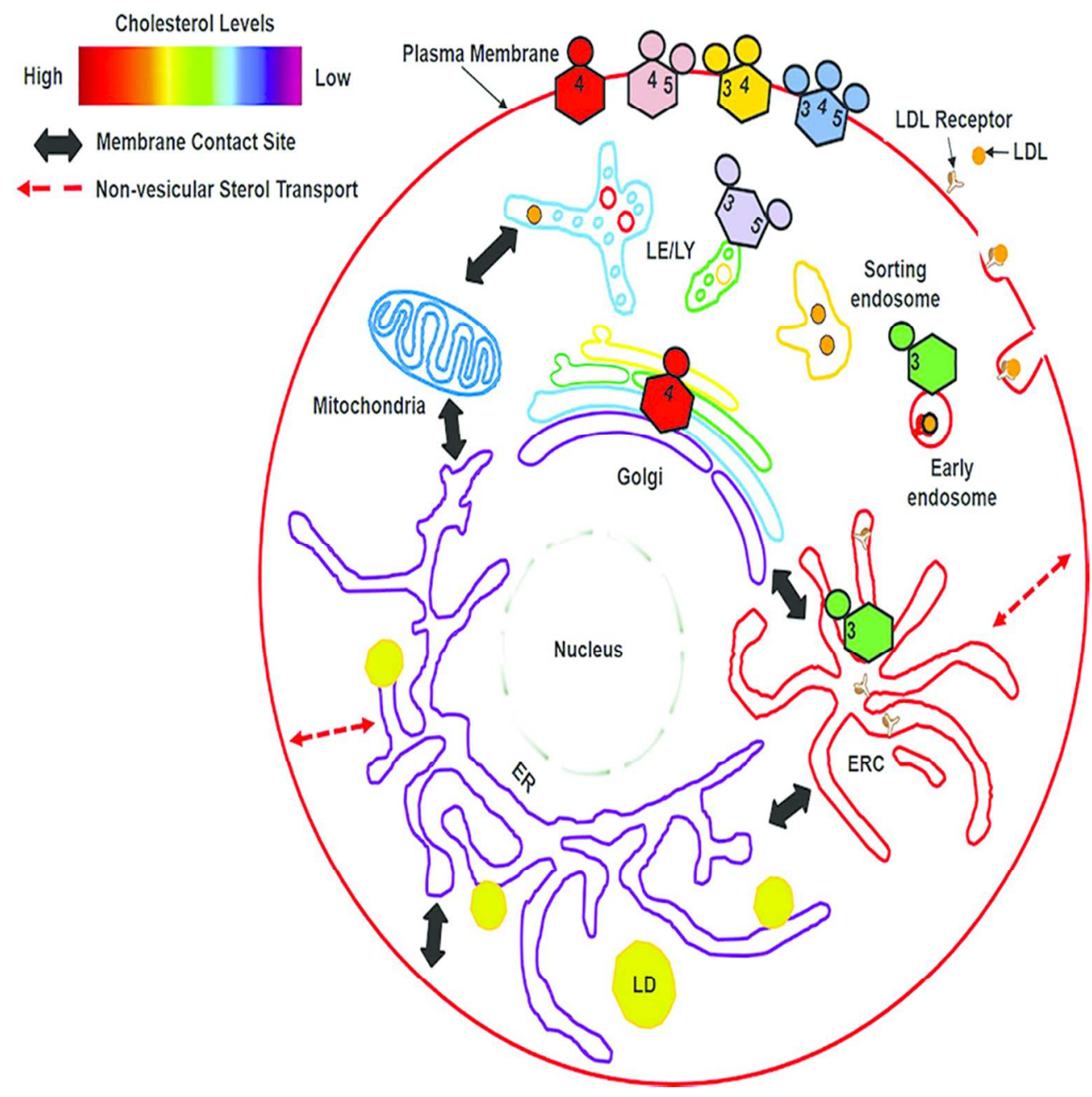

Figure 2: Heterogeneous distribution of cellular cholesterol and phosphatidylinositol phosphates. The cholesterol content of membranes varies among cellular organelles. Membrane cholesterol levels are displayed as a heat map with membranes enriched in cholesterol labeled red. Membrane compartments with relatively low cholesterol levels are labeled blue. The inset describes the transbilayer distribution of cholesterol at the plasma membrane (Lange et al. 1981; Mesmin and Maxfield 2009). Phosphoinositides serve as organelle identity molecules (Balla 2013; Di Paolo and De Camilli 2006): PI3P (green), phosphatidylinositol-3- phosphate; PI4P (red), phosphatidylinositol-4-phosphate; PI5P (purple), phosphatidylinositol-5-phosphate; PI(3,5)P2 (lavender), phosphatidylinositol-(3,5)-bisphosphate; PI(3,4)P2 (gold), phosphatidylinositol-(3,4)-bisphosphate; PI(4,5)P2 (pink), phosphatidylinositol-(4,5)-bisphosphate; and $\mathrm{PI}(3,4,5) \mathrm{P} 3$ (blue), phosphatidylinositol-(3,4,5)-trisphosphate. $86 \times 86 \mathrm{~mm}(300 \times 300 \mathrm{DPI})$ 


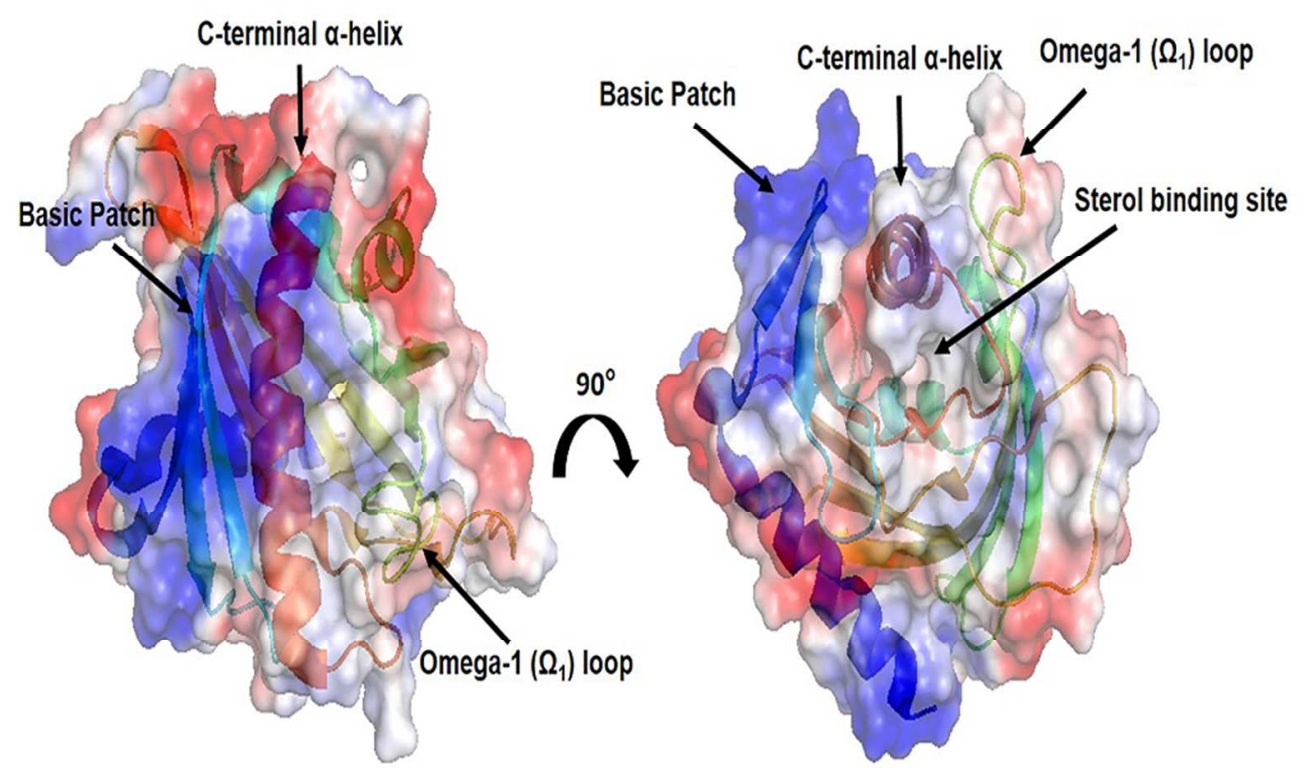

Figure 3: Regions of STARD4 involved in membrane interaction and sterol transport. Surface representation of STARD4 colored according to the electrostatic potential (Iaea et al. 2015). The basic patch, C-terminal ahelix, Omega-1 ( $\Omega 1$ ) loop and sterol binding site are identified in the structures. Right, the structure is rotated $90^{\circ}$ about the $z$-axis for a view of the sterol binding pocket of STARD4.

$86 \times 50 \mathrm{~mm}(300 \times 300$ DPI $)$ 